\title{
Quality evaluation of spaceborne SiC mirrors (I): analytical examination of the effects on mirror accuracy by variation in the thermal expansion property of the mirror surface
}

\author{
Masaki Kotani,, ${ }^{1, \star}$ Tadashi Imai, ${ }^{2}$ Haruyoshi Katayama, ${ }^{2}$ Yukari Yui, ${ }^{2}$ Yoshio Tange, ${ }^{2}$ \\ Hidehiro Kaneda, ${ }^{3}$ Takao Nakagawa, ${ }^{4}$ and Keigo Enya ${ }^{3}$ \\ ${ }^{1}$ Aerospace Research and Development Directorate, Japan Aerospace Exploration \\ Agency, 6-13-1 Ohsawa, Mitaka-shi, Tokyo 181-0015, Japan \\ ${ }^{2}$ Space Applications Mission Directorate, Japan Aerospace Exploration Agency, \\ 2-1-1 Sengen, Tsukuba-shi, Ibaraki 305-8505, Japan \\ ${ }^{3}$ Graduate School of Science, Nagoya University, Furo-cho, Chikusa-ku, Nagoya-shi, Aichi 464-8602, Japan \\ ${ }^{4}$ Institute of Space \& Astronautical Science, Japan Aerospace Exploration Agency, 3-1-1 Yoshinodai, Chuo-ku, \\ Sagamihara-shi, Kanagawa 252-5210, Japan \\ *Corresponding author: kotani.masaki@jaxa.jp
}

Received 26 April 2013; revised 29 May 2013; accepted 29 May 2013; posted 31 May 2013 (Doc. ID 189570); published 3 July 2013

\begin{abstract}
The Japan Aerospace Exploration Agency has studied a large-scale lightweight mirror constructed of reaction-bonded silicon carbide-based material as a key technology in future astronomical and earth observation missions. The authors selected silicon carbide as the promising candidate due to excellent characteristics of specific stiffness and thermal stability. One of the most important technical issues for large-scale ceramic components is the uniformity of the material's property, depending on part and processing. It might influence mirror accuracy due to uneven thermal deformation. The authors conducted systematic case studies for the conditions of CTE by finite element analysis to know the typical influence of material property nonuniformity on mirror accuracy and consequently derived a comprehensive empirical equation for the series of CTE's main factors. In addition, the authors computationally reproduced the mirror accuracy profile of a small prototype mirror shown in cryogenic testing and hereby verified wide-range practical computational evaluation technology of mirror accuracy. (C) 2013 Optical Society of America

OCIS codes: (160.4670) Optical materials; (220.4840) Testing; (240.6700) Surfaces; (350.4600) Optical engineering; (350.6090) Space optics.

http://dx.doi.org/10.1364/AO.52.004797
\end{abstract}

\section{Introduction}

Astronomical and earth observations performed using space telescopes have become increasingly popular in recent years. Space observation has many

$1559-128 \mathrm{X} / 13 / 204797-09 \$ 15.00 / 0$

(C) 2013 Optical Society of America advantages. Spaceborne observation avoids the effects of weather and image fluctuations due to atmospheric flow. It also enables surveillance of gamma rays, X-rays, ultraviolet rays, and far-infrared rays, which offers us more information than Earth-based observations, which are extremely difficult to use for this because of the rays' absorption in the atmosphere. Earth observations from space allow a wide 
field of view and enable us to choose a global-scale observation such as scanning the entire globe or constantly observing a specific region in real time.

Observation satellites have an on board reflecting telescope system such as the Cassegrain reflector as their mission equipment. The primary mirror is the heaviest component in the system, and its weight increases significantly when it is enlarged to enhance the performance of the system. Because of this, as the aperture size is increased, weight-saving measures from the viewpoint of area density (weight of the mirror body per unit area of the mirror surface) is more severely needed. Moreover, the general tendency of manufacturing quality is that as the size (volume) of a product increases, more nonuniformity is likely to occur because of unevenness in thermal conditions and raw materials. Hence, when the aperture size is increased, adequate quality control is needed to avoid deterioration in optical performance and other qualities that can be caused by any nonuniformity. Therefore, to further improve the performance of telescope systems for new missions in the future, it is vital to have the technology to reduce the weight and increase the size of primary mirrors without compromising other qualities and the technology to design, manufacture, and quality-control mirror bodies to support such systems [1,2].

Even though the mirror surface is highly accurate post manufacture, accuracy deteriorates because of thermal deformations occurring during the missions. These deformations happen because the missions operate at a temperature different from the manufacturing temperature and because the satellites are exposed to fluctuations in the temperature while orbiting the Earth. Hence, maintaining mirror accuracy while its body temperature changes is an important quality for the mirror. A small coefficient of thermal expansion (CTE) for a component is more advantageous because a smaller CTE means a smaller amount of deformation in the entire mirror body. However, even with a large CTE, if the deformation of the entire mirror body is uniform, the body is evaluated as high quality.

Amid growing demands in recent years for lighter weight and larger size mirrors, the use of silicon carbide $(\mathrm{SiC})$ has been attracting a lot of attention [3-7]. The infrared astronomical satellite ASTRO$\overline{\mathrm{F}}$ (A $\mathrm{A}$ KARI) was launched in 2006 with the primary mirror constructed of a porous $\mathrm{SiC}$ core coated with chemical-vapor-deposited $\mathrm{SiC}$ equipped onboard [8]. BOOSTEC (France) and EADS Astrium developed the $\mathrm{SiC}$ material "SiC 100" using a sintering process [9], and its segmented primary mirror with a $3.5 \mathrm{~m}$ aperture was equipped on the Herschel Space Observatory and launched in 2009 [10]. Engineered Ceramic Materials GmbH (ECM) (Germany) and Mitsubishi Electric Corporation (Japan) developed the short carbon fiber reinforced $\mathrm{C} / \mathrm{SiC}$ material "HBCesic" by using reaction bonding [11]. This material is the prospective candidate for the primary $3.5 \mathrm{~m}$ dia. mirror to be equipped on the Space
Infrared Telescope for Cosmology and Astrophysics (SPICA) scheduled for launch in 2022 [12,13].

$\mathrm{SiC}$ is a promising material from the viewpoint of providing high specific stiffness (the ratio of Young's modulus to specific weight) and adequate thermal stability (the ratio of thermal conductivity to CTE). $\mathrm{SiC}$ materials have about twice as much specific stiffness and about the same thermal stability when compared to low-thermal expansion glass materials [14]. However, the CTE of SiC materials $\left(2 \times 10^{-6} / \mathrm{K}\right.$ or higher) is more than an order of magnitude greater than that of glass materials $\left(1 \times 10^{-6} / \mathrm{K}\right.$ or less $)$. Thus, in order to secure the same mirror accuracy in a thermally static condition, for example, $\mathrm{SiC}$ mirror bodies must have a higher thermal homogeneity than those made of glass. Hence, to apply $\mathrm{SiC}$ materials, it becomes important to reduce heterogeneitycausing factors during manufacturing and minimize CTE variation in each mirror body part.

In this study, to acquire an evaluation technology to control the quality of $\mathrm{SiC}$ mirror bodies, we conducted an analytical examination of the effect of CTE variation in a mirror's body on the mirror's accuracy to understand its typical behaviors. Using a mirror body that was manufactured and evaluated as a model mirror, we developed an analysis technique that used the finite element method (FEM) to evaluate mirror accuracy in orders of nanometers when deformation of the mirror surface occurred. By using this method, we conducted a case study on the major factors of CTE variation. To verify the effectiveness of the result, we computationally reproduced the experimental result of an actual mirror body by applying the result of the case study. By understanding how each factor affects mirror accuracy, we can clearly define the quality requirements of mirror body materials necessary for obtaining the required mirror accuracy. We will also be able to predict a certain level of mirror accuracy of alreadymanufactured mirror materials by testing extracted specimens without performing mirror polishing and thermal environment mirror measurement, which are enormously time-consuming and costly.

\section{Experimental Methods}

\section{A. Model Mirror Body}

A small prototype mirror made of $\mathrm{SiC}$ was used as a model mirror body to evaluate the typical behavior of a $\mathrm{SiC}$ mirror surface. A photo and schematic of the mirror are shown in Figs. 1 and 2, respectively. The mirror is spherical with an outer diameter of $160 \mathrm{~mm}$ and a focal distance of $454 \mathrm{~mm}$ and is comprised of a mirror skin and a ribbed backside construction. It was manufactured by NEC Toshiba Space Systems, Ltd. using the reaction sintering method [15]. The material was developed as the commercial name "NT-SiC". Heating sintering is a typical method to produce $\mathrm{SiC}$ by the reaction of a pre-molded carbon (C) green body with molten silicon (Si) impregnated at high temperature. 

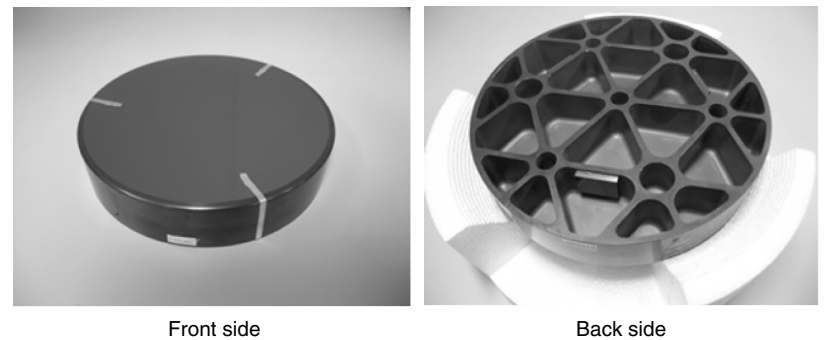

Fig. 1. Prototype $\mathrm{SiC}$ mirror comprised of a mirror skin and a ribbed backside construction. Left, front side; Right, back side.

Compared to other manufacturing methods, it has an advantage in increasing the size of mirrors, an important aspect of mirror body development in the future. Cryogenic testing on the mirror was conducted to evaluate how a precooled mirror surface changed its mirror accuracy by cooling [16]. Three fiducial points attached on the rim of the mirror surface, as shown in Fig. 1, were small pieces of aluminum tape, which were used to subtract the surface figure measured at a room temperature from those at cryogenic temperatures. This study covered the entire mirror body, including the ribbed construction, and we evaluated the effect on mirror accuracy attributed to the difference of thermal expansion properties between one part of the mirror surface and the others.

\section{B. Analysis of Mirror Accuracy}

As shown in Fig. 3, the entire surface of the mirror body was divided into 19 regular hexagons, with opposite sides $30 \mathrm{~mm}$ distant (an area of approx. $780 \mathrm{~mm}^{2}$ each), on the orthogonal projection of the mirror body. One of the regions (marked "9" in Fig. 3) was given a different CTE from the other regions. The temperature of the entire mirror body was uniformly changed from 300 to $100 \mathrm{~K}$. A series of operations, from creation of finite element (FE) models, setting of boundary conditions, calculation of FEM analysis to output of the analysis result, was carried out by using the general purpose analysis code NEiNastran V9.1 SOL. 101 of NEi Software, Inc. (USA).

An FE model of the mirror body shown in Fig. 4 was created. The whole model was comprised of solid elements with 218,900 nodes and 179,927 elements. In order to examine the effect of the size of regions with different material properties, a separate FE model was created where regular hexagonal regions with the opposite sides $15 \mathrm{~mm}$ distant $\left(195 \mathrm{~mm}^{2}\right)$, $6 \mathrm{~mm}$ distant $\left(31 \mathrm{~mm}^{2}\right)$, and $3 \mathrm{~mm}$ distant $\left(7.8 \mathrm{~mm}^{2}\right)$ were placed concentrically inside Region 9 , as shown in Fig. 5. With 223,068 nodes and 183,988 elements, the scale of the second model was similar to that of the original model. Segmented regions of both models, including the ribbed structure, were given the same material property in the direction of the normal to the center of the mirror surface.

Boundary conditions shown in Fig. $\underline{6}$ were set to both FE models. In order to fix the base position of the mirror surface, all three translations in the $x$, $y$, and $z$ directions at the mirror surface center were constrained. In order to restrict the tilt of the mirror surface around the point, the translations in the $x$ and $y$ directions at the center of the mirror skin at the back were constrained. The translation in the $z$ direction was not constrained here in order to deal with the change in the thickness of the mirror skin caused by thermal deformation. Finally, in order to restrict the rotation around the central axis of the mirror body, the translation of the $y$ direction at both edges of the mirror surface in the direction of the $x$ axis from the center of the mirror surface and the translation of the $x$ direction at both edges of the mirror surface in the direction of the $y$ axis from the center of the mirror surface, were constrained. In this way, the motion of the mirror body was constrained symmetrically without interfering with the thermal deformation of the mirror body.

Then, various material properties and the temperature change from 300 to $100 \mathrm{~K}$ were applied to the analytical models, and computation was performed using the aforementioned software. This gave the displacement distribution of the mirror surface caused by the thermal deformation of the mirror body. However, a contour diagram of the mirror surface at this stage may strongly include inclination- or radius-dependent components, rendering the evaluation of mirror accuracy difficult. Therefore, in order to eliminate these elements as much as possible, post treatment was applied by substituting the coordinates $\left(x_{i}, y_{i}, z_{i}\right)$ of all the nodes on the mirror surface after deformation into Eq. (1) of the spherical surface with center $(a, b, c)$ and radius $r_{i}$. The values $R, a, b$, and $c$, which minimized the residual sum of squares
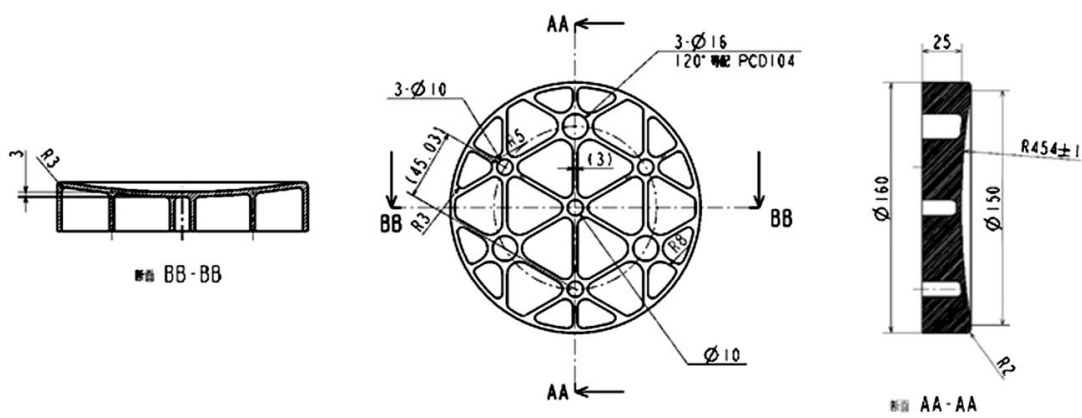

Fig. 2. Drawing of a prototype mirror. Spherical mirror surface with an outer diameter of $160 \mathrm{~mm}$ and a focal distance of $454 \mathrm{~mm}$. 


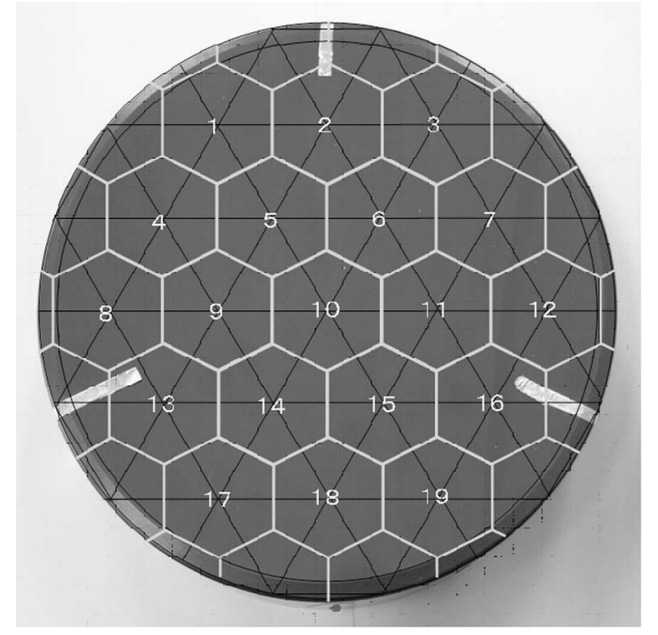

Fig. 3. Nineteen orthogonally projected segmented regions on the mirror surface used in this examination. The regions are regular hexagons with opposite side distances of $30 \mathrm{~mm}$ and an area of $\sim 780 \mathrm{~mm}^{2}$ each.
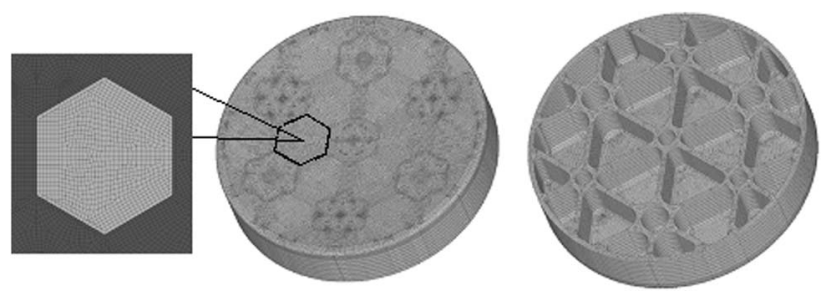

Fig. 4. Finite element model of the mirror body entirely comprised of solid elements with 218,900 nodes and 183,988 elements.

$J$ computed from Eq. (2), were derived by using the nonlinear least-squares method:

$$
\begin{gathered}
r_{i}(a, b, c)=\sqrt{\left(x_{i}-a\right)^{2}-\left(y_{i}-b\right)^{2}-\left(z_{i}-c\right)^{2}}, \\
J=\sum_{i=1}^{n}\left(R-r_{i}(a, b, c)\right)^{2} .
\end{gathered}
$$

$R$ and $(a, b, c)$, obtained here are the radius and the coordinates of the center of the spherical surface that best fits the mirror surface after deformation. These

(a)

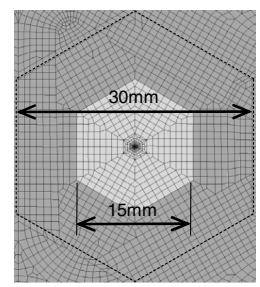

(b)

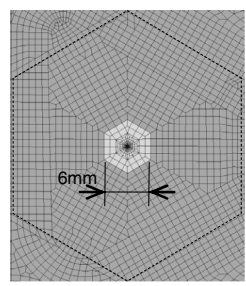

Fig. 5. Specific regions used to examine the effect of the size of regions with different material properties. Those were placed concentrically inside the Region 9. Opposite side distances: (a) $15 \mathrm{~mm}$, (b) $6 \mathrm{~mm}$, and (c) $3 \mathrm{~mm}$.

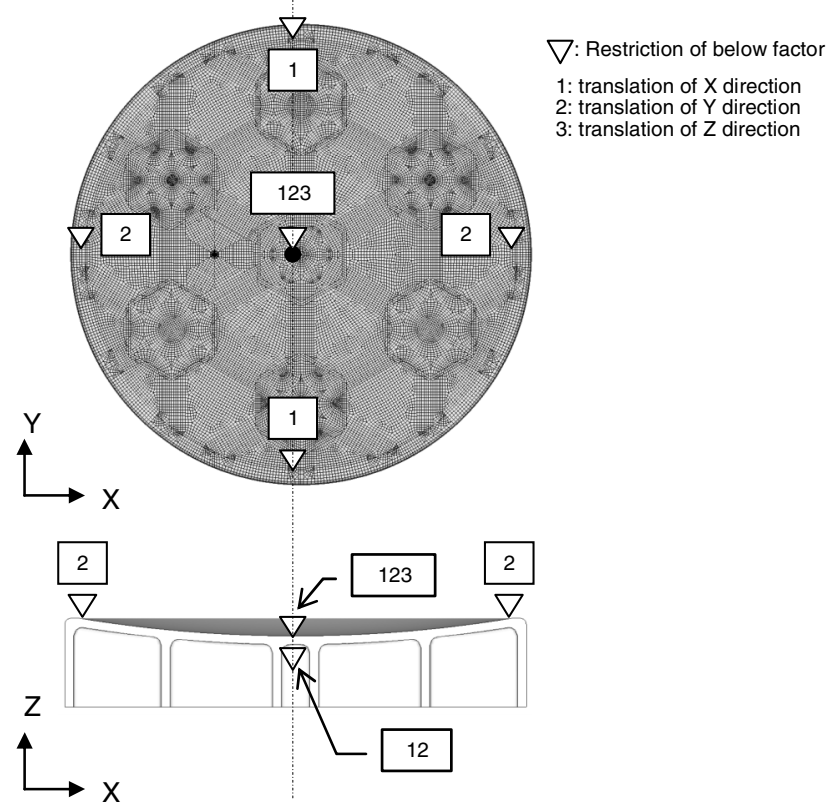

Fig. 6. Boundary conditions set to the FE models. Necessary motion constraints were symmetrically given.

were used as the focus and radius of curvature of the spherical mirror surface, which represented the nodes after deformation. The distribution of deviations from the spherical mirror surface was expressed in a contour diagram, from which the "deviation from the representation spherical surface" was found by picking up numerical values and computing [(Representative value in the periphery of the region with different CTE)-(Representative value inside the region with different CTE)]. Deviations in the direction approaching the focus were given a positive sign, and the result was used to evaluate mirror accuracy.

\section{Material property}

In the analysis, the values that had been obtained from experimenting on a test piece that was made of the material manufactured under the same conditions as the mirror body were used for the base values of the material properties. Those are shown together with other representative $\mathrm{SiC}$ mirror materials in Table $\underline{1}[14,15]$. Input CTE, which was measured at room temperature, was used as the average CTE value of the component in the range from 100 to $300 \mathrm{~K}$. Elastic modulus and Poisson's ratio were also measured at room temperature. When the CTE of a segmented region and that of the other regions differed by $+\mathrm{X} \%$ and $-\mathrm{X} \%$ (or $-\mathrm{X} \%$ and $+\mathrm{X} \%$ ) from the base value, the case was called "the case of $2 \mathrm{X} \%$ (or $-2 \mathrm{X} \%$ ) difference."

First of all, in order to verify the validity of whether the above-mentioned analysis method was effective in evaluating mirror accuracy in orders of nanometers, we conducted an analysis under the condition that the entire mirror body had the same material property values. After verifying the validity, 
Table 1. Base Values of the Material Properties used in the FEM Analysis [15] ${ }^{a}$

\begin{tabular}{lcccccc}
\hline Material & $\begin{array}{c}\mathrm{CTE} \\
\left(\times 10^{-6} / \mathrm{K}\right)\end{array}$ & $\begin{array}{c}\text { Elastic Modulus } \\
(\mathrm{GPa})\end{array}$ & $\begin{array}{c}\text { Poisson's } \\
\text { Ratio }\end{array}$ & $\begin{array}{c}\text { Density } \\
\left(\mathrm{g} / \mathrm{cm}^{3}\right)\end{array}$ & $\begin{array}{c}\text { Specific Heat } \\
(\mathrm{J} / \mathrm{kg} / \mathrm{K})\end{array}$ & $\begin{array}{c}\text { Thermal Conductivity } \\
(\mathrm{W} / \mathrm{m} / \mathrm{K})\end{array}$ \\
\hline NT-SiC (used in this & 2.4 & 360 & 0.18 & 3.02 & 677 & 130 \\
study) & & & & & & \\
SiC (RB-30\% Si) & 2.64 & 310 & & 2.92 & 660 & 158 \\
SiC (RB-12\% Si) & 2.68 & 373 & & 3.11 & 680 & 147 \\
SiC (CVD) & 2.4 & 466 & 0.21 & 3.21 & 700 & 146 \\
CESIC & 2.6 & 235 & & 2.65 & 660 & $\sim 135$ \\
\hline
\end{tabular}

${ }^{a}$ Table involves representative $\mathrm{SiC}$ mirror materials data [14].

we conducted a case study about the major factors of the nonuniformity of the thermal expansion property by changing the CTE of a part of the mirror surface. The major factors we focused on were: (i) the "base CTE value", which is the fundamental value possessed by the component, (ii) the rate of CTE variation that may exist depending on the location in the mirror body, and (iii) the size of the region in which CTE varies. Factor (i) depends on the material used (including its manufacturing method and conditions), and Factors (ii) and (iii) are directly associated with manufacturing quality, which is attributed to the nonuniformity factors in the raw materials or the manufacturing process. After that, in order to verify that the case study result of the partial mirror surface was applicable to the evaluation of the mirror accuracy of the entire mirror surface, we tried to reproduce the result of the cryogenic testing by inputting the material property values, which were calculated back from the case study result, into segmented regions of the mirror surface.

\section{Results and Discussion}

\section{A. Verification of the Method of Analysis}

We conducted the analysis when an identical CTE value was input into the entire mirror body and consequently obtained the contour diagram showing the distribution of deviations of the mirror surface from the representation mirror surface after thermal deformation, which is viewed from the focus of the representation surface. The maximum difference [peak to valley (PV) value] of the radius of curvature of each part was $30 \mathrm{~nm}$. The magnitude of this value was comparable to the numerical error arising from software's digit-number constraints on the node coordinate values. Thus, we concluded that this analysis method was valid for evaluating mirror accuracy from the distribution of material properties, as long as the resulting values exceeded the magnitude of the aforementioned numerical error.

An example of the analysis result in the case where only one segmented region of the mirror surface had a different CTE from the other regions is shown in Fig. 7 . The left figure is the contour diagram before post treatment and the right figure is the one after post treatment. They are, from left to right, the distribution of deviations from the original spherical surface and the distribution of deviations from the representation spherical surface after deformation. In this example, in which the CTE of a segmented region was smaller than that of the other areas, this part looks like it is approaching the focus in comparison to the areas around it. In the left figure, the values are generally declining as areas move away from the focus from the left-hand to right-hand side, but in the right figure, in which the decline was averaged out, it is easier to distinguish the deviations occurring in the segmented region.

\section{B. Effect of Base CTE}

For the case where the CTE of a segmented region and that of the other regions differed from the base value by $+0.5 \%(-0.5 \%)$ and $-0.5 \%(+0.5 \%)$

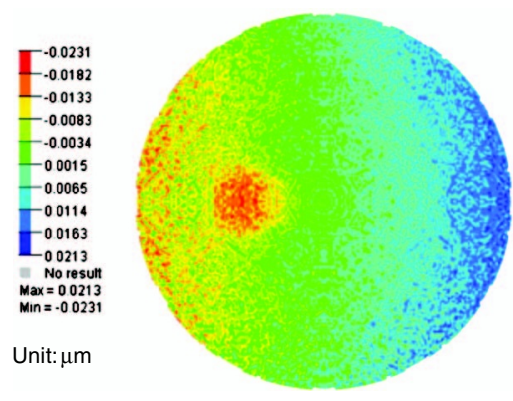

Before post treatment

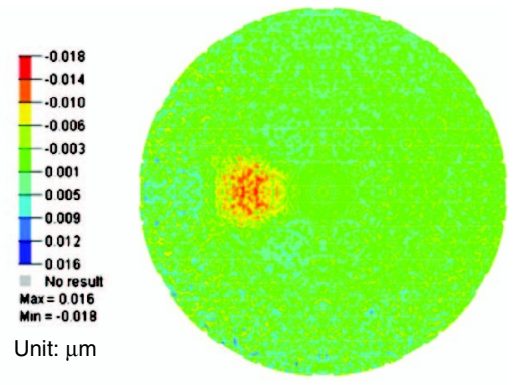

After post treatment

Fig. 7. Example of the analysis result in the case where only one segmented region of the mirror surface had a different CTE from the other regions (left: the distribution of deviations from the original spherical surface; right: the distribution of deviations from the representation spherical surface). Base CTE: $2.4 \times 10^{-6} / \mathrm{K}$, CTE variation: $0.1 \%$, opposite side distance: $30 \mathrm{~mm}^{(a r e a: ~} \sim 780 \mathrm{~mm}{ }^{2}$, and temperature: $300 \rightarrow 100 \mathrm{~K}$. 
respectively, (i.e., the case of $1 \%$ difference), the analysis result of deviations from the representation spherical surface obtained from various base CTEs is shown in Fig. 8. In the segmented region, when its CTE was smaller than that of the surrounding regions, deviations approaching toward the focus were generated, and conversely, when its CTE was larger, deviations moving away from the focus were generated. The absolute magnitude of deviation (hereinafter called "the magnitude of deviation") was about the same at the same base CTE. It increased linearly with respect to the base value. When the slope of the approximating straight line passing through the origin was computed by using the leastsquares method, it was 0.047 in each case. This means that the base CTE of the mirror body component linearly affects the magnitude of deviation of the mirror surface, which is caused by CTE variation, and that when the CTE of an area of about $780 \mathrm{~mm}^{2}$ is $1 \%$ smaller than the CTE of surrounding regions, the deviation of about $47 \mathrm{~nm}$ toward the focus is generated per base CTE of $1 \times 10^{-6} / \mathrm{K}$. As mentioned earlier, glass materials $\left(1 \times 10^{-6} / \mathrm{K}\right.$ or less $)$ have an advantage at this point over $\mathrm{SiC}$ materials $\left(2 \times 10^{-6} / \mathrm{K}\right.$ or higher $)$.

\section{Effect of the Rate of CTE Variation}

Based on the CTE value given in Table 1 $\left(2.4 \times 10^{-6} / \mathrm{K}\right)$, for the case when the CTE of a segmented region and that of the other regions differed at various rates with opposite signs, the analysis result of deviations from the representation spherical surface is shown in Fig. 9. This figure also includes the results of the cases in which the region with different CTE had opposite sides at a distance other than $30 \mathrm{~mm}$. As seen in the previous section, when the CTE of the segmented region was smaller (larger) than that of the other regions, deviations approaching toward (moving away from) the focus were generated. In the same-sized region, the magnitude of

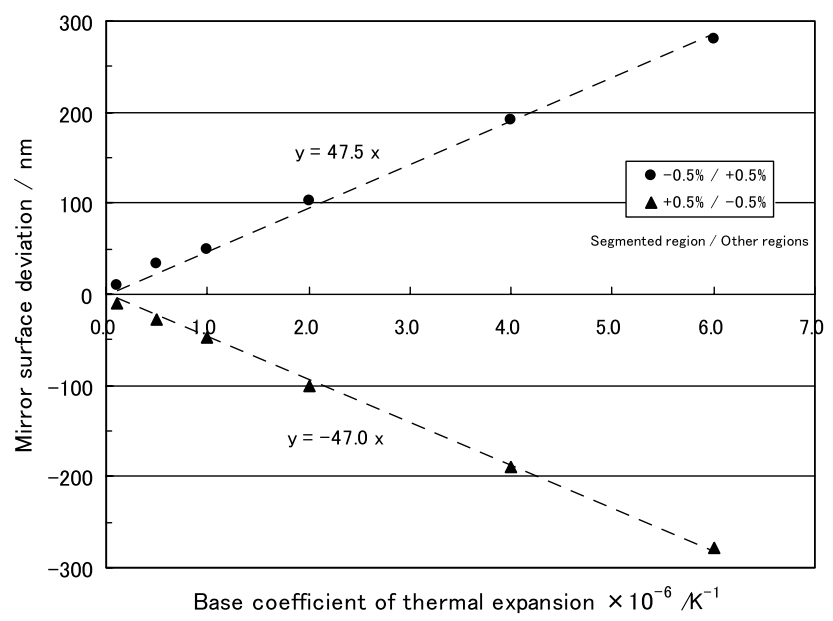

Fig. 8. Analysis result of deviations from the representation spherical surface obtained from various base CTEs. CTE variation: $1 \%$, opposite side distance: $30 \mathrm{~mm}$ (area: $\sim 78 \mathrm{~mm}^{2}$ ), and temperature: $300 \rightarrow 100 \mathrm{~K}$.

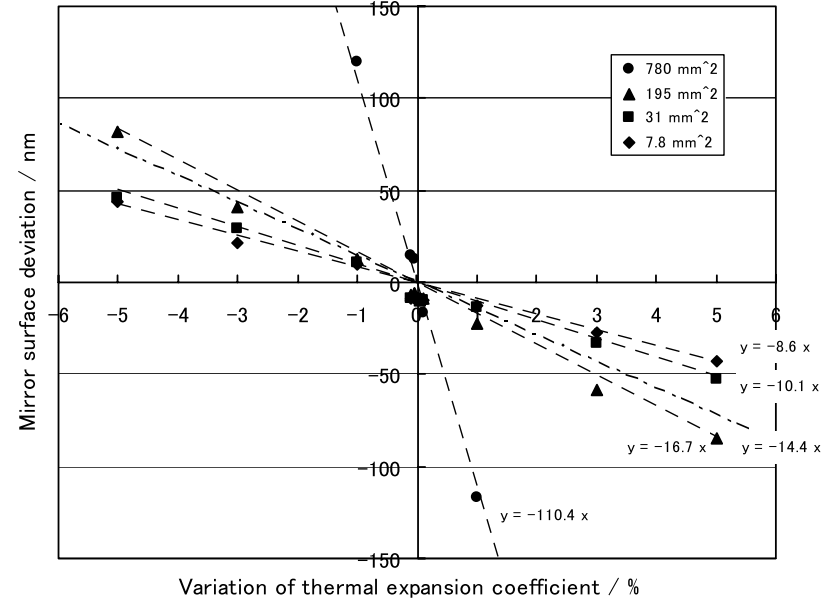

Fig. 9. Analysis result of deviations from the representation spherical surface at various rates of CTE variation. Base CTE: $2.4 \times 10^{-6} / \mathrm{K}$, and temperature: $300 \rightarrow 100 \mathrm{~K}$.

deviation increased linearly with respect to the rate of CTE variation in all cases. The slope of the approximating straight line passing through the origin was computed in cases with different region sizes by using the least-squares method. This result is also shown in Fig. 9. From this result, it can be seen that the rate of CTE variation of the mirror body component linearly affected the mirror surface deviation, and that with the base CTE of $2.4 \times 10^{-6} / \mathrm{K}$, when the CTE of an area of about $780 \mathrm{~mm}^{2}$ was smaller than the CTE of surrounding regions, the deviation of about $110 \mathrm{~nm}$ toward the focus was generated per $1 \%$ variation of the CTE.

\section{Effect of the Size of the Region in which CTE is Varied}

The effect of the size of the region in the mirror body in which CTE was varied was observed first in the relation between the dashed-dotted curve, which was only associated with thermal deformation of the material, and each of the analysis results (Fig. 9). This dashed-dotted curve indicates the difference in thickness that is expected to be generated when two independent pieces of material with the same thickness as the mirror skin $(3 \mathrm{~mm})$ and with the same CTEs as the segmented region and the other regions, respectively, go through the same temperature change as in the analysis $(300 \rightarrow 100 \mathrm{~K})$. These are equivalent to the deviations of the mirror surface, which occur only on the surface side, are caused only by material properties, and are unaffected by any other parts. When its magnitude of deviation was compared with that of a region with the same rate of CTE variation, the analysis result when opposing sides were $30 \mathrm{~mm}$ distant was significantly larger than this result; the result when opposing sides were $15 \mathrm{~mm}$ distant was close to this result, and the results of 6 and $3 \mathrm{~mm}$ were smaller than this result. Opposing sides that were several times the mirror skin thickness became the border value. Any region larger than that had a mirror surface deviation 
larger than the difference occurring between two independent pieces due to the effect of the surrounding area, and any region smaller than that had the mirror surface deviation smaller than the difference of two independent pieces. In particular, when the distance between opposing sides was more than several times the mirror skin thickness, the deviation became significantly larger than the difference of two independent pieces. The reason for this was that the mirror skin was distorted by the interference of the surrounding area and deformed outward. On the other hand, the reason for the smaller deviation was probably because the difference in thickness affected not only the mirror surface side but also the backside of the skin.

The analysis result shown in Fig. 9 was sorted with respect to the areas and opposite side distances of the segmented regions with different CTEs, and the sorted results were shown in Figs. 10 and 11, respectively. In both cases, with the same rate of $\overline{\mathrm{CT}} \mathrm{E}$ variation, the magnitude of deviation tended to increase at an accelerating rate as the region became larger. In particular, the initial inclination of the curve for opposite side distances was small compared with that of the area, but subsequently its inclination increased at an accelerating rate. When the approximating curves for the area and the opposite side distances were computed, it was found that the area and the opposite side distance behaved like a quadratic polynomial curve and a cubic polynomial curve, respectively. In the case where the magnitude of CTE variation (the absolute value of the rate of CTE variation) remained the same and only the magnitude relation of the segmented region and the other regions was reversed, their magnitudes of deviation stayed about the same, and thus, we concluded that this effect of the magnitude relation was negligible. By finding approximating curves to the average value of the magnitude of deviation in the cases of $-1 \%$ and $+1 \%$ in Figs. 10 and 11, we obtained estimated magnitudes of deviation in terms of the

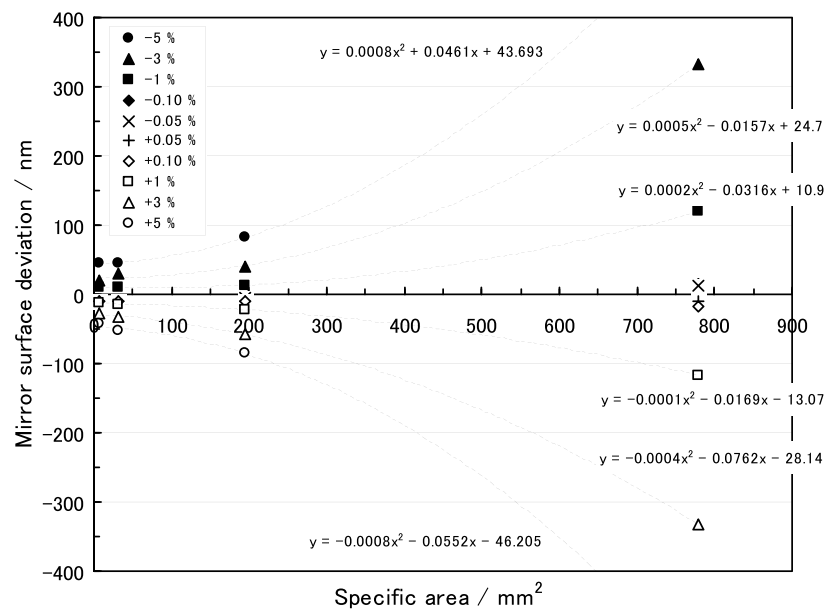

Fig. 10. Analysis result of deviations from the representation spherical surface at various areas of the specific region. Base CTE: $2.4 \times 10^{-6} / \mathrm{K}$, temperature: $300 \rightarrow 100 \mathrm{~K}$.

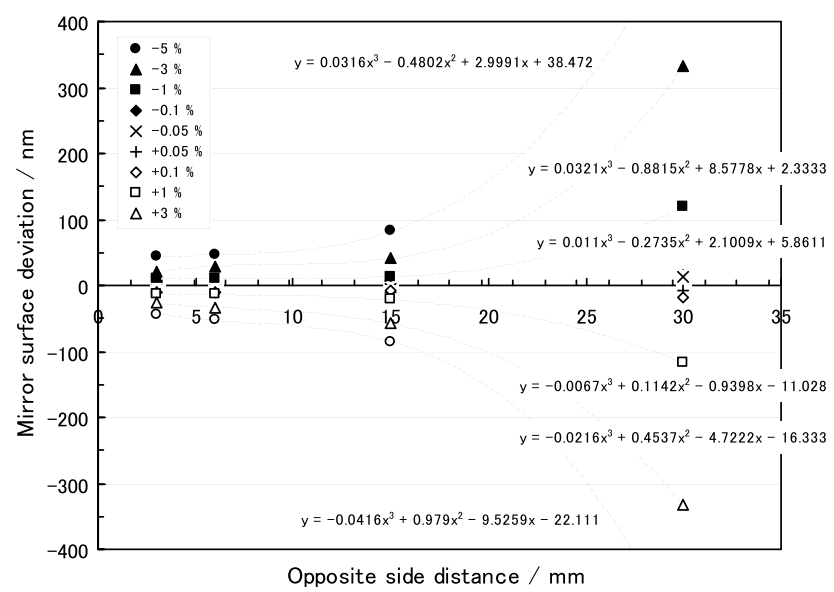

Fig. 11. Analysis result of deviations from the representation spherical surface at various opposite side distances of the specific region. Base CTE: $2.4 \times 10^{-6} / \mathrm{K}$, temperature: $300 \rightarrow 100 \mathrm{~K}$.

area $S\left(\mathrm{~mm}^{2}\right)$ and the opposite side distance $\underline{d}(\mathrm{~mm})$, respectively: $0.0002 S^{2}-0.0074 S+12.007(\mathrm{~nm})$ and $0.0088 d^{3}-0.1938 d^{2}+1.5204 d+8.444(\mathrm{~nm})$. This means that the size of the region with different CTEs on the mirror surface nonlinearly affects the magnitude of deviation of the mirror surface. Also, when the CTE of a segmented region is $1 \%$ smaller than the CTE of surrounding areas, with the base CTE of $2.4 \times 10^{-6} / \mathrm{K}$, the deviation close to the one estimated by the formulas earlier in the paragraph, toward the focus, is generated.

\section{E. Formulation of the Magnitude of Deviation Caused by Major Factors}

From Sections 3.B to 3.D, we have described individual effects of three major factors of CTE variation on mirror surface deviations. By combining these results, we can now consider a formula for estimating mirror surface deviations under any given conditions of these three factors. So far we have found that (1) the base CTE and the rate of CTE variation affect deviation linearly, and the deviation is estimated to be about $47 \mathrm{~nm}$ toward the focus in an area of about $780 \mathrm{~mm}^{2}$ per base CTE of $1 \times 10^{-6} / \mathrm{K}$ and CTE variation of $-1 \%$; and (2) the area and distance between opposing sides affect deviation nonlinearly, and the deviations are estimated to be about $0.0002 S^{2}-0.007 S+12(\mathrm{~nm})$ and $0.009 d^{3}-0.2 d^{2}+$ $1.5 d+8.4(\mathrm{~nm})$ toward the focus, respectively, per base CTE of $2.4 \times 10^{-6} / \mathrm{K}$ and CTE variation of $-1 \%$ per $1 \mathrm{~mm}^{2}$ and $1 \mathrm{~mm}$, respectively. Therefore, with the deviation toward the focus of the mirror surface denoted as $D(\mathrm{~nm})$, the base CTE as $a\left(\mathrm{~K}^{-1}\right)$, and the rate of CTE variation as $r(\%)$, the formulas for estimating the mirror surface deviation with respect to the area of the specific region $S\left(\mathrm{~mm}^{2}\right)$ and the distance between opposing sides $d(\mathrm{~mm})$ were presumably written as the following Eqs. (ㅁ) and ()ㅜㄴ:

$$
\begin{aligned}
D & =-a \cdot 10^{6} \cdot r \cdot\left(0.0002 S^{2}-0.007 S+12\right) \div 2.4 \\
& =-417000 \cdot a \cdot r \cdot\left(0.0002 S^{2}-0.007 S+12\right),
\end{aligned}
$$




$$
\begin{aligned}
D & =-a \cdot 10^{6} \cdot r \cdot\left(0.009 d^{3}-0.2 d^{2}+15 d+8.4\right) \div 2.4 \\
& =-417000 \cdot a \cdot r \cdot\left(0.009 d^{3}-0.2 d^{2}+15 d+8.4\right) .
\end{aligned}
$$

By using these formulas, the mirror surface deviation can be estimated with an arbitrary combination of the three major factors, and the estimated value provides basic information that is helpful for selecting a material for developing the desired mirror body, setting the permissible range of manufacturing quality, and estimating the quality of a manufactured material as a mirror body component. The applicable range of the conditions of individual factors and other factors associated with mirror accuracy should be considered as needed.

\section{F. Verification of the Effectiveness of the Case Study's Result}

It is necessary to verify that the case study's result for a part of the mirror surface is applicable to the accuracy evaluation of the entire mirror surface. For this purpose, we tried to reproduce the result of the cryogenic mirror's accuracy testing of the actual mirror body by applying the material conditions based on the case study result to the entire mirror body model. The analysis was conducted by reading the high-low values corresponding to the individual segmented regions on the mirror surface from the test result, computing the CTE value to generate these deviations from the correlation between mirror surface deviation and CTE value found in the case study, and inputting the value into each region.

The result of the cryogenic testing of the actual mirror body is shown in Fig. 12. The result was obtained by the Institute of Space and Astronautical Science of Japan Aerospace Exploration Agency (JAXA) by measuring the difference in mirror accuracy with a He-Ne laser (632.8 nm wavelength) when the mirror body was uniformly cooled from 300 to $95 \mathrm{~K}$ [16]. Wave numbers corresponding to the colors at the center position of the 19 segmented

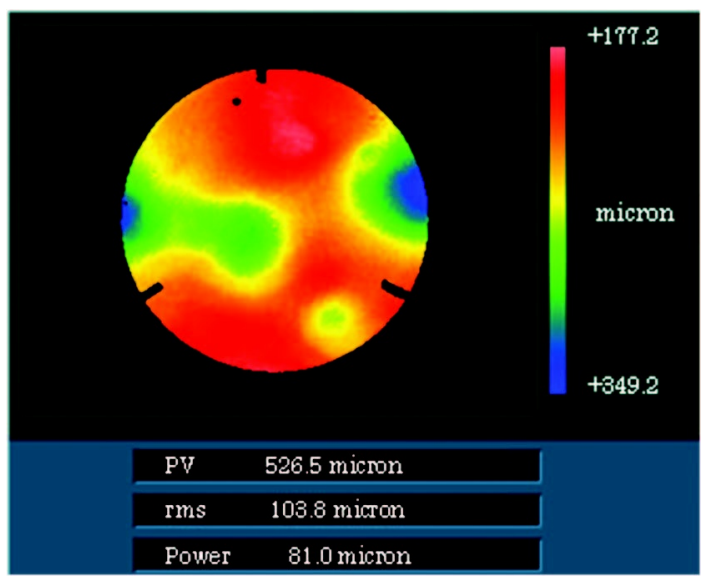

Fig. 12. Result of the cryogenic mirror accuracy testing of the actual mirror body. Temperature: $300 \rightarrow 95 \mathrm{~K}, \mathrm{PV}=526 \mathrm{~nm}$ [16]. regions were read from the contour bar, and values of the mirror surface deviation of these regions were calculated by multiplying their wave numbers by the wavelength of the $\mathrm{He}-\mathrm{Ne}$ laser $(632.8 \mathrm{~nm})$. Finally, the CTE values that caused these deviations were found from the case study result in Fig. $\underline{9}$ and used for evaluation of the analysis. The resulting contour figure is shown in Fig. 13. In general, the figure looks quite similar to the test result obtained from the experiment. The PV value $(453 \mathrm{~nm})$ was relatively close to the test result ( $526 \mathrm{~nm})$. Thus, the reproduction can be described as a success.

Therefore, it was verified that the result of the case study, which was focused on one part of the mirror surface, was valid at a certain level for the evaluation of the mirror accuracy of the entire mirror surface. This means that if we can use the result of the case study as a database to find the CTE distribution conditions of the mirror surface, we can predict to a certain degree how the mirror surface deviation of the mirror body will be distributed. Conversely, from the distribution of a mirror surface deviation that a certain mirror body shows in an experiment, we can predict to a certain degree what the CTE distribution of the mirror surface will be like. This allows us to evaluate the quality of a sintered block, which is manufactured under certain conditions using certain equipment, as a mirror body material by simply testing sample pieces without mirror processing, which normally takes longer than several months. Moreover, when the result of the mirror surface test of a certain mirror body is available and if the relation between its material structure and thermal expansion property is known, we can predict what the distribution of thermal expansion property of the mirror body will be like, and, if necessary, the information can be fed back to the manufacturing technology to reduce the nonuniformity of the material structure, thus improving quality.

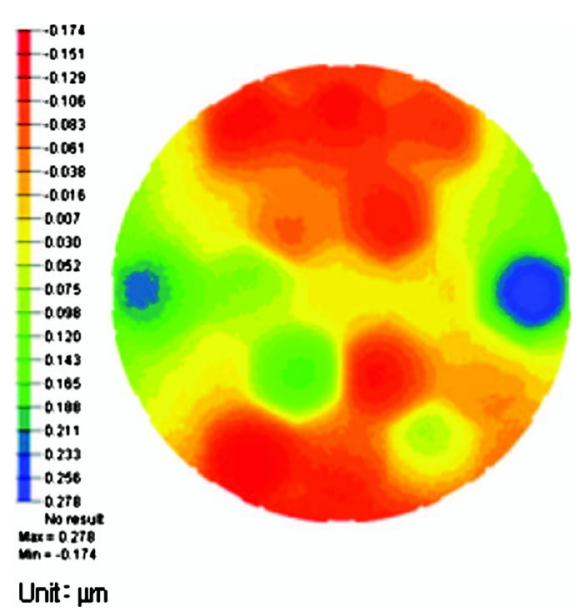

Fig. 13. Contour figure obtained by the FEM analysis for reproducing the result of the cryogenic testing of the actual mirror body. CTE values inputted in the FE model were based on the result of the case study in Fig. $\underline{9}$. 


\section{Conclusion}

Using a model SiC mirror body, we conducted an analysis evaluation with FE models that divided a mirror surface into small regions and examined the effect of the variation in the thermal expansion property of the mirror body on mirror accuracy. Major results obtained from the study are summarized below.

1. We constructed an evaluation method for mirror accuracy that was comprised of FEM analysis using general-purpose software and a spherical surface-fitting process on the nodes on the mirror surface, and verified its effectiveness.

2. We quantitatively summarized how the base values of CTE, the degrees of CTE variation, and the size of the region affected mirror-surface deviation when a CTE variation existed on a part of the mirror surface. We also showed that the base CTE value and the degrees of CTE variation linearly affected deviation and that the size of a region affected deviation at an accelerating rate. We derived formulas to predict mirror surface deviation by comprehensively combining these findings.

3. By reproducing the result of the cryogenic mirror accuracy testing of the actual mirror body, we verified that the mirror surface deviation data of a part of the mirror surface was valid for evaluating the mirror accuracy of the entire mirror surface.

4. We saw that the result obtained in this study quantitatively correlates mirror accuracy with the CTE distribution conditions of the mirror body. This finding will greatly help improve the efficiency of the development of the mirror body, where increasingly higher accuracy and larger size are in demand.

The authors acknowledge the various support provided by Mr. Yoshikazu Muta of Tokyo University of Science for examinations in the early stage of the study, and by JAXA staff Ms. Rei Ueda and Mr. Kenichi Tomoda for the processing of the analysis data. We also acknowledge the support provided by Mr. Soichi Takeda and Mr. Keisuke Arafurue of Digital Solutions Inc. in various aspects of mirror accuracy analysis. We would like to take this opportunity to express our gratitude for all of your support and guidance.

\section{References}

1. R. Edeson, G. S. Aglietti, and A. R. L. Tatnall, "Conventional stable structures for space optics: the state of the art," Acta Astronaut. 66, 13-32 (2010).

2. J. W. Bilbro, "Lightweight mirrors/optical materials," in Encyclopedia of Modern Optics, R. D. Guenther, G. D. Steel, and L. Bayvel, eds. (Elsevier, 2005), pp. 460-466.

3. J. S. Goela and R. L. Taylor, "Large scale fabrication of lightweight Si/SiC lidar mirrors," Proc. SPIE 1118, 14-24 (1989).

4. R. A. Paquin, M. B. Magida, and C. L. Vernold, "Large optics from silicon carbide," Proc. SPIE 1618, 53-60 (1991).

5. Y. Zhang, J. Zhang, J. Han, X. He, and W. Yao, "Large-scale fabrication of lightweight $\mathrm{Si} / \mathrm{SiC}$ ceramic composite optical mirror," Mater. Lett. 58, 1204-1208 (2004).

6. E. Sein, Y. Toulemont, J. Breysse, P. Deny, D. Chambure, T. Nakagawa, and M. Hirabayashi, "A new generation of large $\mathrm{SiC}$ telescopes for space applications," Proc. SPIE 5528, 83 (2004).

7. J. Breysse, D. Castel, B. Laviron, D. Logut, and M. Bougoin, "All-SiC telescope technology: recent progress and achievements," in Proceedings of the 5th International Conference on Space Optics (ICSO 2004), B. Warmbein, ed. (ESA, 2004), pp. 659-671.

8. H. Kaneda, T. Onaka, and R. Yamashiro, "Development of SiC mirror for ASTRO-F," The Institute of Space and Astronautical Science Report 14, 289-295 (2000).

9. D. Logut, J. Breysse, Y. Toulemont, and M. Bougoin, "Light weight monolithic silicon carbide telescope for space application," Proc. SPIE 5962, 59621Q (2005).

10. S. Y. Emmanuel, F. S. Toulemont, D. Michel, D. Pierre, D. C. Daniel, P. Thomas, and P. Göran, "A $\phi 3.5 \mathrm{~m} \mathrm{SiC}$ telescope for Herschel mission," Proc. SPIE 4850, 606-618 (2003).

11. M. R. Krödel and T. Ozaki, "HB-cesic composite for space optics and structures," Proc. SPIE 6666, 66660E (2007).

12. T. Nakagawa, and SPICA Working Group, "SPICA: space infrared telescope for cosmology and astrophysics," Adv. Space Res. 34, 645-650 (2004).

13. T. Onaka and T. Nakagawa, "SPICA: a $3.5 \mathrm{~m}$ space infrared telescope for mid- and far-infrared astronomy," Adv. Space Res. 36, 1123-1127 (2005).

14. R. Y. Paul, Jr., Opto-mechanical Systems Design, 3rd ed. (SPIE, 2006), pp. 111-112.

15. K. Tsuno, H. Irikado, K. Oono, J. Ishida, S. Suyama, Y. Itoh, N. Ebizuka, H. Eto, Y. Dai, W. Lin, T. Suzuki, H. Omori, Y. Y. Yui, T. Kimura, and Y. Tange, "New-technology silicon carbide (NT-SiC): demonstration of new material for large lightweight optical mirror," Proc. SPIE 5659, 138-145 (2005).

16. H. Kaneda, T. Nakagawa, T. Onaka, K. Enya, H. Kataza, S. Makiuti, H. Matsuhara, M. Miyamoto, H. Murakami, H. Saruwatari, H. Watarai, and Y. Y. Yui, "Development of lightweight SiC Mirrors for the space infrared telescope for cosmology and astrophysics (SPICA) mission," Proc. SPIE 6666, 666607 (2007). 